\title{
Shallow pockets and very strong coupling superconductivity in $\mathrm{FeSe}_{x} \mathrm{Te}_{1-x}$
}

\author{
Y. Lubashevsky, E. Lahoud, K. Chashka, D. Podolsky and A. Kanigel ${ }^{\star}$
}

The celebrated Bardeen-Cooper-Schrieffer (BCS) theory has been successful in explaining metallic superconductors, yet many believe that it must be modified to deal with the newer high-temperature superconductors. A possible extension is provided by the BCS-Bose-Einstein condensate (BEC) theory', describing a smooth evolution from a system of weakly interacting pairs to a BEC of molecules of strongly bound fermions. Despite its appeal, spectroscopic evidence for the BCS-BEC crossover has never been observed in solids. Here we report electronic structure measurements in $\mathrm{FeSe}_{x} \mathrm{Te}_{1-x}$ showing that these materials are in the BCS-BEC crossover regime. Above the superconducting transition temperature, $T_{c r}$ we find multiple bands with remarkably small values for the Fermi energy $\varepsilon_{\mathrm{F}}$. Yet, in the superconducting state, the gap $\Delta$ is comparable to $\varepsilon_{\mathrm{F}}$. The ratio $\Delta / \varepsilon_{\mathrm{F}} \approx 0.5$ is much larger than found in any previously studied superconductor, resulting in an anomalous dispersion of the coherence peak very similar to that found in cold Fermi gas experiments ${ }^{2}$, in agreement with the predictions of the BCS-BEC crossover theory.

The BCS-BEC theory is based on the observation that the BCS wave function can describe both a system of weakly interacting pairs and a BEC of molecules of strongly bound fermions, provided that the fermion chemical potential is allowed to change sign with increasing interaction strength ${ }^{3,4}$. The crossover is governed by the interplay between pairing interaction, represented by the energy gap $\Delta$, and kinetic energy, represented by the Fermi energy $\varepsilon_{\mathrm{F}}$.

Cold Fermi gasses provide the most direct way to see the BCS-BEC crossover ${ }^{5,6}$ - the Feshbach resonance offers a way to tune the interaction between atoms continuously. As the interaction strength is increased one can observe the change in the chemical potential, $\mu$. It has been shown ${ }^{2}$ that the fermion momentum distribution changes in the way predicted by the BCS-BEC theory ${ }^{1,3,4}$.

Many authors have predicted similar physics in the underdoped cuprates because of their large gaps and low carrier concentration $^{7-9}$. It was suggested that many of their unconventional properties could be explained by the BCS-BEC crossover, but spectroscopic evidence of a change in $\mu$ has never been found. In this paper we look instead at the iron chalcogenide $\mathrm{FeSe}_{x} \mathrm{Te}_{1-x}$ and present evidence for BCS-BEC crossover physics in this system.

We have performed angle-resolved photoemission spectroscopy (ARPES) measurements to map the band structure, both above and below $T_{\mathrm{c}}$. We find a band structure similar to the one reported in previous ARPES work ${ }^{10-12}$. A sketch of the Fermi surface of $\mathrm{FeSe}_{x} \mathrm{Te}_{1-x}$, together with a measured intensity map at $\varepsilon_{\mathrm{F}}$ is shown in Fig. 1 .

ARPES data taken above $T_{\mathrm{c}}$, at $15 \mathrm{~K}$, around the $\Gamma$ point can be seen in Fig. 2. The different orbital character of the bands leads to a strong polarization and orientation dependence of the intensity ${ }^{12}$.

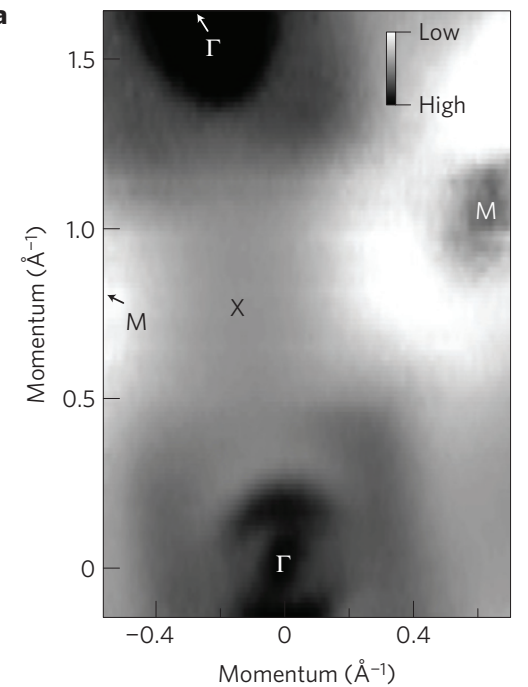

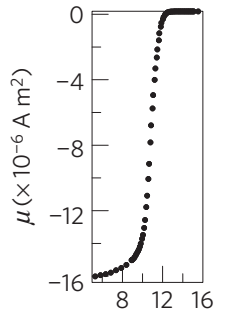

Temperature $(\mathrm{K})$

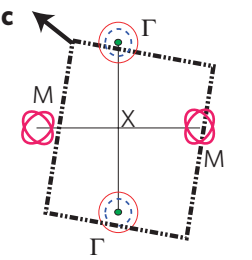

Figure 1 | Fermi surface of $\mathrm{FeSe}_{\mathbf{x}} \mathrm{Te}_{\mathbf{1}-\mathbf{x}} \cdot \mathbf{a}$, Intensity map at the Fermi level integrated over 4 meV. b. Magnetic moment versus temperature, showing a sharp superconducting transition at $12.5 \mathrm{~K}$. c, A sketch of the Brillouin zone of $\mathrm{FeSe}_{x} \mathrm{Te}_{1-x}$.

In Fig. $2 \mathrm{a}$ and $\mathrm{b}$ we show ARPES data and the corresponding momentum distribution curves (MDCs) taken parallel to the $\Gamma-\mathrm{M}$ direction and the $\Gamma-\mathrm{X}$ direction, respectively. Three bands can be identified; we use the notation $\alpha_{1}, \alpha_{2}$ and $\alpha_{3}$ following ref. 10 . We extract the masses and the Fermi crossing points of the different bands by taking many cuts covering a large region around $\Gamma$, see Fig. $2 c$ and d. For each cut we use the MDCs to map out the dispersion. We fit the entire data set using three circular paraboloids. The fit to the data is excellent, see Fig. 2e and $\mathrm{f}$, allowing us to extract the Fermi crossing points and the value of $\varepsilon_{\mathrm{F}}$ for the various bands.

We find that only $\alpha_{2}$ and $\alpha_{3}$ cross the Fermi energy and create hole pockets. $\alpha_{1}$ ends about $25 \mathrm{meV}$ below the Fermi surface. The two bands that form the Fermi surface are strongly renormalized in comparison with the calculated band structure. The two pockets are very shallow; based on the fit we estimate $\varepsilon_{\mathrm{F}}$ to be $4 \pm 2.5 \mathrm{meV}$ for both pockets. For the electron-pocket around the M-point we find $\varepsilon_{\mathrm{F}}$ to be $10 \pm 1 \mathrm{meV}$ (see Supplementary Fig. S1). This is a surprising result: a metal with such a small Fermi energy cannot be considered a degenerate Fermi gas even at room temperature.

Our values for $\varepsilon_{\mathrm{F}}$ are in close agreement with transport measurements ${ }^{13}$ which infer a $T_{\mathrm{F}}$ of $150 \mathrm{~K}$ from the Seebeck coefficient. The remarkable agreement (taking into account that the 


$$
\text { a }
$$

Momentum $\left(\AA^{-1}\right)$

c

d

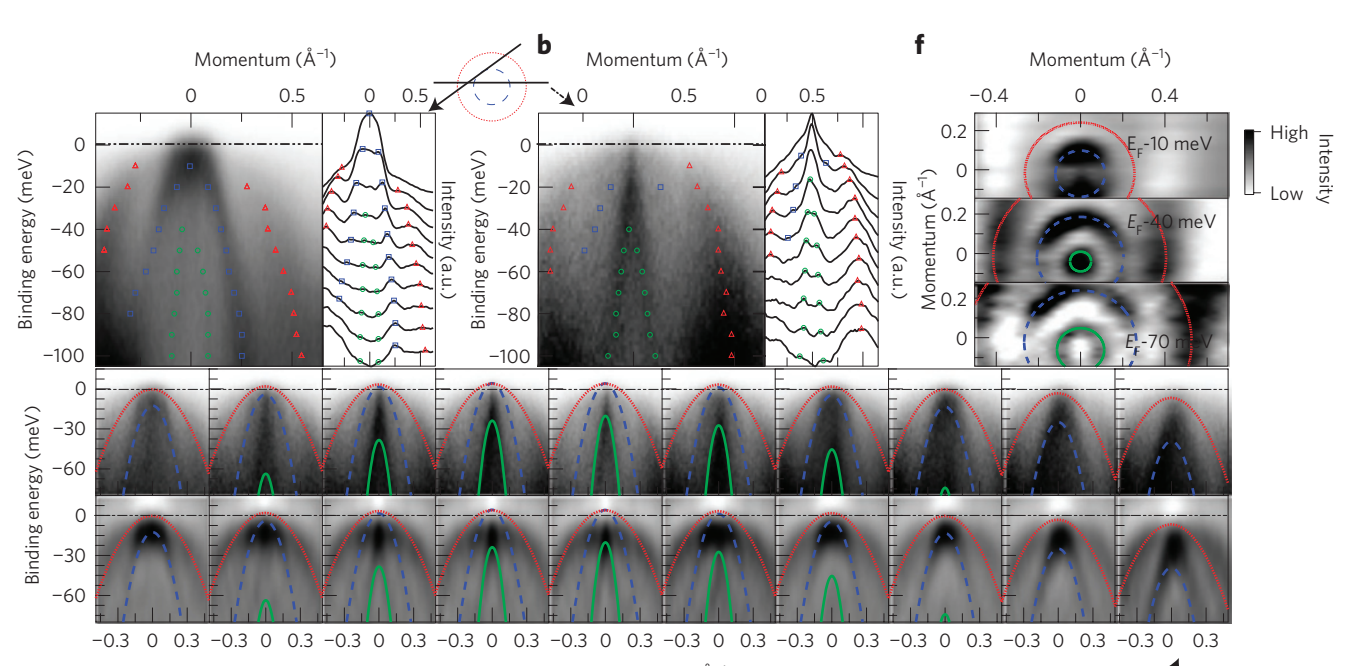

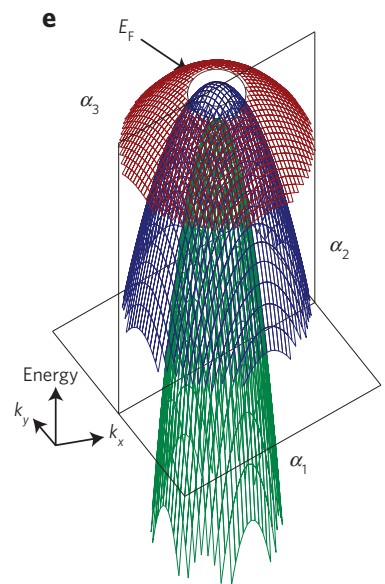

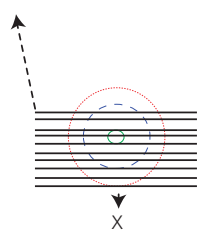

Momentum $\left(\AA^{-1}\right)$

- $\circ \alpha_{1}$

- - $\alpha_{2}$

.... $\Delta \alpha_{3}$

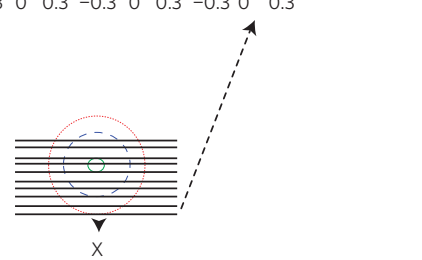

Figure $2 \mid \boldsymbol{\Gamma}$ point data. $\mathbf{a}, \mathbf{b}$, ARPES data and the corresponding MDCs for a cut taken in the $\Gamma-\mathrm{M}(\mathbf{a})$ and $\Gamma-\mathrm{X}(\mathbf{b})$ directions. $\mathbf{c}$, ARPES data for 10 different cuts taken parallel to the $\Gamma-X$ direction. $\mathbf{d}$, Second derivative with respect to energy of the data shown in $\mathbf{c}$. e, The two-dimensional fit results, the three paraboloids are the best-fit to the data representing the three hole-like bands. We find that $\alpha_{1}$ does not crossing the Fermi level. For both $\alpha_{2}$ and $\alpha_{3}$ we find small pockets with $\varepsilon_{\mathrm{F}}=4 \pm 2.5 \mathrm{meV}$. We find the following masses for the different bands: $m_{\alpha_{1}}^{\star}=1.0 \pm 0.3 m_{\mathrm{e}}, m_{\alpha_{2}}^{\star}=3.4 \pm 0.5 m_{\mathrm{e}}$ and $m_{\alpha_{3}}^{\star}=14 \pm 3 m_{\mathrm{e}}$. These values are in good agreement with previous work ${ }^{10}$. f, Intensity maps at three different binding energies, with solid lines taken from the fit.

a
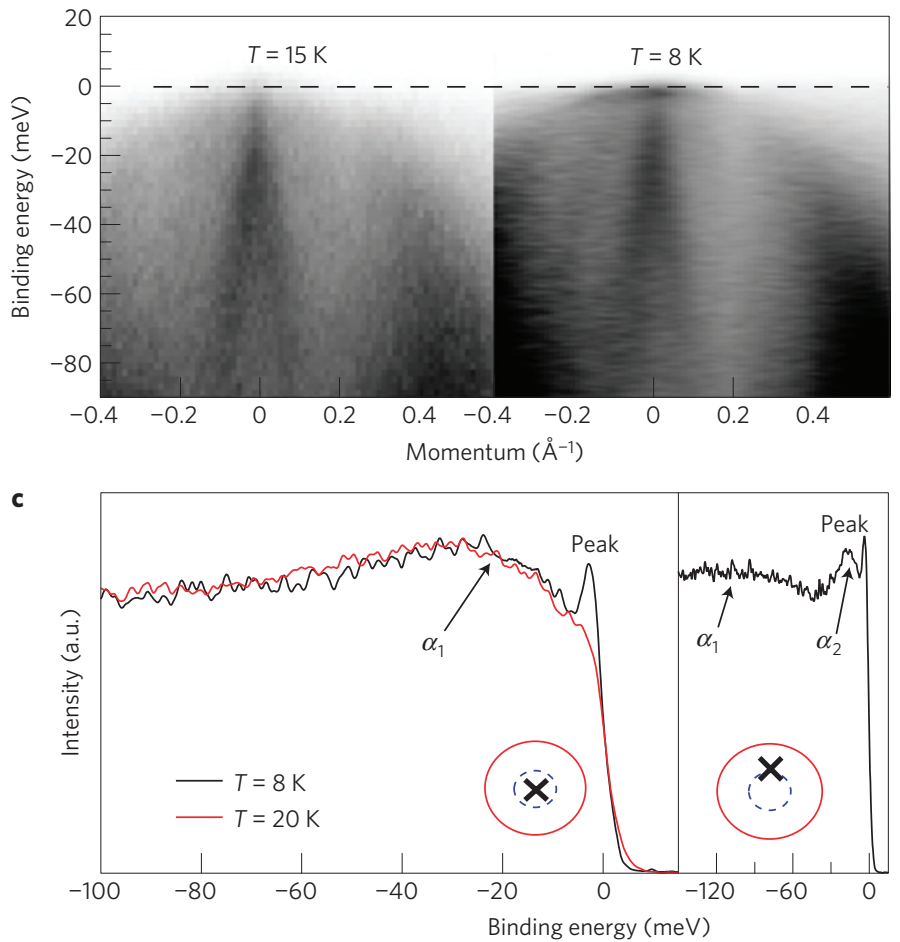

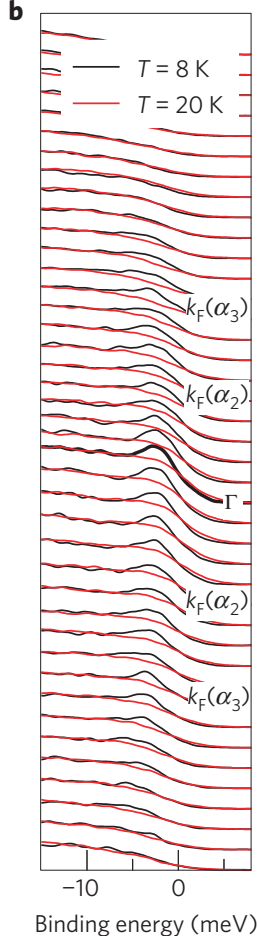

Figure 3 | Coherence peak. a, Detector images from ARPES data going through the $\Gamma$ point taken at $8 \mathrm{~K}$ (right) and $15 \mathrm{~K}$ (left). $\mathbf{b}$, A direct comparison of EDCs above and below $T_{C}$, the coherence peak is clearly visible. The line shape in the superconducting state is similar to the line shape found in the cuprates (see Supplementary Fig. S2). c, A comparison of the EDC above and below $T_{\mathrm{c}}$ at the $\Gamma$ point (left) and the EDC below $T_{\mathrm{c}}$ at a momentum point between the two hole pockets (right).

single-band model used in ref. 13 provides an upper limit of $T_{\mathrm{F}}$ ) between a bulk-sensitive transport measurement and our ARPES results indicates that our results are representative of the bulk.
When we lower the temperature below $T_{\mathrm{c}}$, we find surprising results. In Fig. 3a (right) we show the raw ARPES data of a cut going through the $\Gamma$ point taken below $T_{\mathrm{c}}$. In contrast to the data taken 

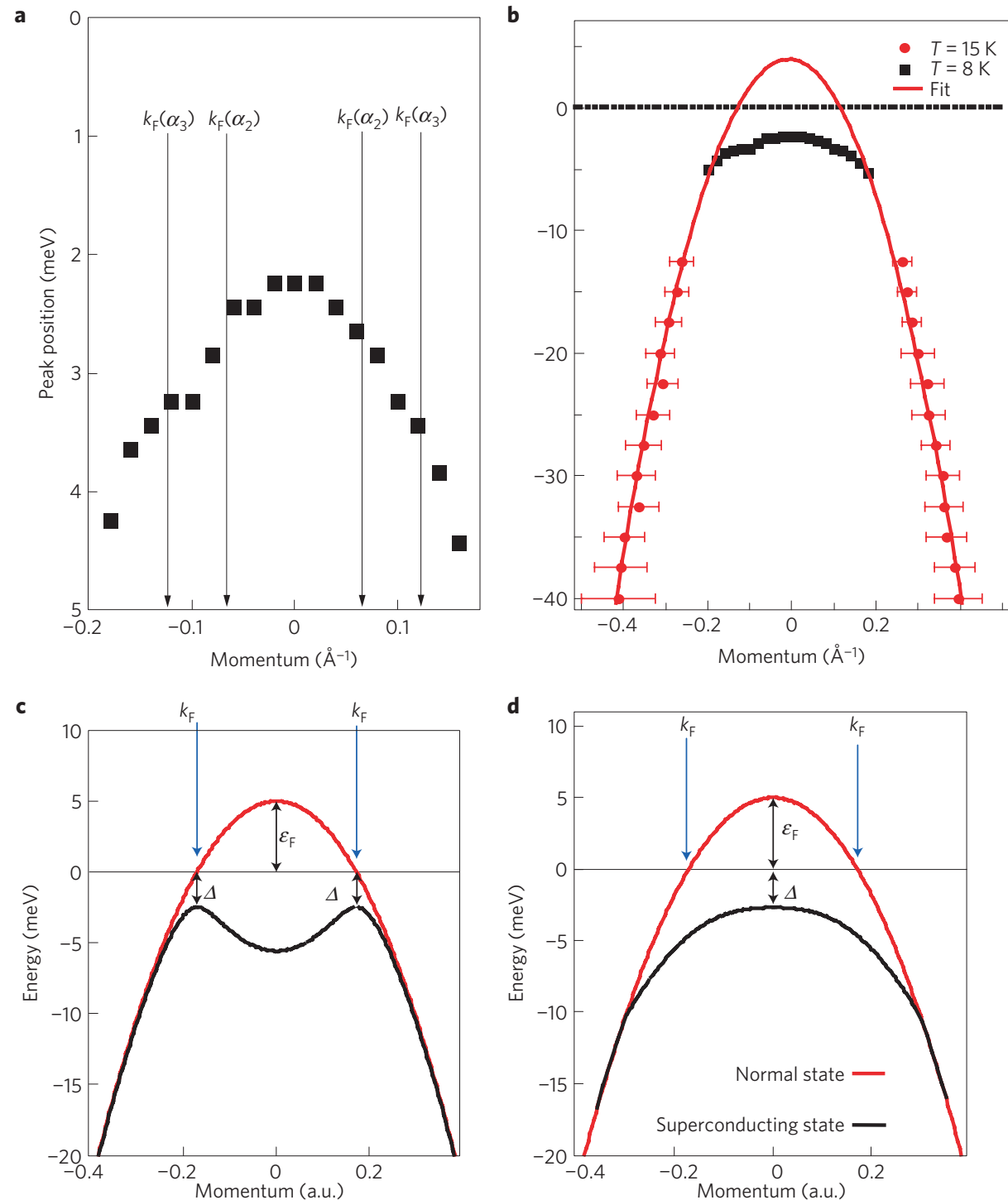

Figure 4 | Dispersion below $T_{\mathrm{c}}$ and the BCS-BEC crossover model. a, Dispersion of the coherence-peak as extracted from the EDCs shown in Fig. $3 \mathrm{~b}$. b. Dispersion of the $\alpha_{3}$ band. The red points represent MDC peak positions, they are found to be the same above and below $T_{\mathrm{c}}$. The black squares represent the coherence peak position. The red line is from the two-dimensional fit. The error bars represent the uncertainty in the MDC peak position obtained by fitting a Gaussian to the MDC peaks. c, A sketch of the quasiparticle dispersion for the case $\tilde{\varepsilon}_{F} \sim \varepsilon_{F}$ (BCS limit). d, A sketch of the quasiparticle dispersion for the case $\tilde{\varepsilon}_{\mathrm{F}}=0$ (BCS-BEC crossover regime).

above $T_{\mathrm{c}}$ (Fig. 3a (left)), one can see the emergence of a coherence peak below $T_{\mathrm{c}}$. In Fig. $3 \mathrm{~b}$ we show a direct comparison of the energy distribution curves (EDCs) measured at $20 \mathrm{~K}$ and at $8 \mathrm{~K}$, the new peak is very sharp, its width is about $3 \mathrm{meV}$ and it is probably resolution-limited. The coherence peak can be found over a large portion of the zone centred around the $\Gamma$ point. In Fig. $3 \mathrm{c}$ (left) we compare the EDC at the $\Gamma$ point above and below $T_{\mathrm{c}}$. There is an 'hump' peaked around $25 \mathrm{meV}$ whose origin is the $\alpha_{1}$ band, the hump position and shape does not depend on temperature. Below $T_{\mathrm{c}}$ there is an extra sharp peak. For comparison we show in Fig. 3c (right) the EDC below $T_{\mathrm{c}}$ at a momentum point which lies between the two Fermi pockets (see inset), where it is possible to identify both $\alpha_{1}$ and $\alpha_{2}$ in addition to the sharp coherence peak. It is possible to follow the coherence peak even beyond $k_{\mathrm{F}}$ of the $\alpha_{3}$ band.

The coherence peak position disperses as one moves from the $\Gamma$ point, as shown in Fig. $4 \mathrm{a}$. In Fig. $4 \mathrm{~b}$ we show the dispersion of the $\alpha_{3}$ band and of the coherence peak. The dispersion of the $\alpha_{3}$ band was extracted from the MDC peak positions. We can follow the band up to $12 \mathrm{meV}$ from $\varepsilon_{\mathrm{F}}$, the same dispersion is found above and below $T_{\mathrm{c}}$ within the error bars shown in the figure. The signal is very weak when approaching $\varepsilon_{\mathrm{F}}$, and it is unlikely that this is attributed to a matrix-element effect because the intensity depends on the binding energy very strongly. This is a pseudogap, which is expected in the normal state when the interaction is strong enough ${ }^{14}$. The strong interactions broaden all the features, making it impossible to follow the dispersion in the pseudogap state up to $\varepsilon_{\mathrm{F}}$.

The dispersion below $T_{\mathrm{c}}$ was extracted by following the peak position in the EDCs. Remarkably, the minimal gap is found exactly at the $\Gamma$ point, and not around $k_{\mathrm{F}}$. In fact, nothing special happens at $k_{\mathrm{F}}$, as can be seen in Fig. 4a. The coherence peak dispersion reveals a very flat dispersion below $T_{\mathrm{c}}$, and to relate the dispersion of the coherence peak to that of $\alpha_{3}$ we must conclude that the effective mass is strongly renormalized when the sample becomes superconducting.

The gap size at the $\Gamma$ point is $\Delta=2.3 \pm 0.3 \mathrm{meV}$; the zero temperature gap should be slightly larger. Comparing that to $\varepsilon_{\mathrm{F}}$, we get $\Delta / \varepsilon_{\mathrm{F}} \sim 0.5$. This is a large number, indicating strong-coupling 
superconductivity in $\mathrm{FeSe}_{x} \mathrm{Te}_{1-x}$. For comparison $\Delta / \varepsilon_{\mathrm{F}} \sim 0.1$ in optimally doped Bi2212.

In the BCS theory, below $T_{\mathrm{c}}$ the quasiparticle dispersion is given by $E_{\mathrm{k}}= \pm \sqrt{\xi_{\mathrm{k}}^{2}+\Delta_{\mathrm{k}}^{2}}$, where $\Delta_{\mathrm{k}}$ is the gap function and $\xi_{\mathrm{k}}= \pm\left(\varepsilon_{\mathrm{k}}-\tilde{\varepsilon}_{\mathrm{F}}\right)$ is the band dispersion measured relative to a banddependent shift $\tilde{\varepsilon}_{\mathrm{F}}$. Here, $\varepsilon_{\mathrm{k}}=k^{2} / 2 m^{*}$, and the sign of $\xi_{\mathrm{k}}$ depends on whether the band is electron-like or hole-like. For a single band, it is conventional to replace $\tilde{\varepsilon}_{\mathrm{F}}$ by the chemical potential $\mu$ within the crossover mean-field theory. In the current context, however, we prefer to use $\tilde{\varepsilon}_{\mathrm{F}}$, as each band has its own Fermi energy, and to allow for the independent renormalization of the Fermi energy of the bands, for example due to self-energy effects below $T_{\mathrm{c}}$ (ref. 15).

To find $\tilde{\varepsilon}_{\mathrm{F}}$ and $\Delta_{\mathrm{k}}$ one needs to solve the BCS gap and number equations self-consistently ${ }^{16}$. In the weak-coupling limit $\tilde{\varepsilon}_{\mathrm{F}}=\varepsilon_{\mathrm{F}}$ to an excellent approximation and we get the Bogolyubov dispersion with the characteristic back-bending of the occupied branch. The dispersion is given by $E_{\mathrm{k}}=-\sqrt{\varepsilon_{\mathrm{k}}^{2}+\Delta^{2}}$, where the energy is measured relative to $\varepsilon_{\mathrm{F}}$. The weak-coupling situation for a hole-like band is shown in Fig. 4c. In this case the minimum of the energy gap occurs at $k_{\mathrm{F}}$ and its value is $\Delta$. At $k=0$ the quasiparticle energy is $\sqrt{\varepsilon_{\mathrm{F}}^{2}+\Delta^{2}}$. This characteristic dispersion was measured using ARPES in the cuprates ${ }^{17}$ and in the pnictides ${ }^{18}$.

On the other hand, when the interaction is strong enough, there is a significant shift in $\tilde{\varepsilon}_{\mathrm{F}}$. As $\tilde{\varepsilon}_{\mathrm{F}}$ becomes smaller, the position where the gap minimum is found shifts away from the normal state $k_{\mathrm{F}}$ towards $k=0$. In the extreme case, where $\tilde{\varepsilon}_{\mathrm{F}}$ reaches zero (or changes sign), the minimum of the gap moves to the $\Gamma$ point and its value becomes $E_{\Gamma}=-\sqrt{\tilde{\varepsilon}_{\mathrm{F}}^{2}+\Delta^{2}}$ (ref. 1). This quasiparticle dispersion as predicted by the model in the case where $\tilde{\varepsilon}_{\mathrm{F}}$ is exactly zero is shown in Fig. $4 \mathrm{~d}$. The similarity to our data, shown in Fig. $4 \mathrm{~b}$, is striking.

We find $\Delta / \varepsilon_{\mathrm{F}}=0.6 \pm 0.4$. This estimate is based on the assumption that $\tilde{\varepsilon}_{\mathrm{F}}$ is close to zero. Although we cannot determine $\Delta$ and $\tilde{\varepsilon}_{\mathrm{F}}$ independently, our data is inconsistent with a very negative $\tilde{\varepsilon}_{\mathrm{F}}$, as that would lead to a much larger binding energy at the $\Gamma$ point than the normal state $\varepsilon_{\mathrm{F}}$. On the other hand, if the change in $\tilde{\varepsilon}_{\mathrm{F}}$ were small, the minimum of the binding energy below $T_{\mathrm{c}}$ would be at $k_{\mathrm{F}}$ instead of at the $\Gamma$ point. This indicates that our sample does not lie deep in either the BCS or BEC regimes, but lies instead in the crossover regime.

In its simplest form, the BCS-BEC crossover theory is in good qualitative agreement with our data, although it does not give a full quantitative description. In particular, the mean-field theory predicts that for a two-dimensional system the gap must be twice as large as $\varepsilon_{\mathrm{F}}$ in order for $\tilde{\varepsilon} s_{\mathrm{F}}$ to change sign on crossing $T_{\mathrm{c}}$ (ref. 19). To get quantitative agreement one may need to include multiple bands, momentum-dependent interactions, and self-energy corrections, which play an important role in renormalizing the band structure.

\section{Methods}

For the ARPES experiments we have grown high-quality single crystals of $\mathrm{FeSe}_{x} \mathrm{Te}_{1-x}$ using the 'self-flux' method ${ }^{20}$. Energy-dispersive spectroscopy shows a uniform composition with $x=0.35$. $T_{\mathrm{c}}$ of these crystals is $12.5 \mathrm{~K}$, as found using superconducting quantum interference device (SQUID) magnetometry (Fig. 1b). The transition is sharp $\left(\Delta T_{\mathrm{c}}<1 \mathrm{~K}\right)$ and the magnetic moment size indicates a large superconducting volume fraction of about $100 \%$ at low temperatures.

The ARPES measurements were done at the Technion, using a Scienta R4000 and the $\mathrm{HeI}_{\alpha}$ line $(21.218 \mathrm{eV})$ from a He lamp. Crystals of typical size of $1 \times 1 \mathrm{~mm}^{2}$ were cleaved in situ at a pressure lower than $5 \times 10^{-11}$ torr. The samples were cleaved at $8 \mathrm{~K}$, and measured below and above $T_{\mathrm{c}}$.
Received 14 April 2011; accepted 22 December 2011; published online 5 February 2012

\section{References}

1. Randeria, M. in Bose-Einstein Condensation (eds Griffin, A., Snoke, D. W. \& Stringari, S.) 355-392 (Cambridge Univ. Press, 1995).

2. Stewart, J. T., Gaebler, J. P. \& Jin, D. S. Using photoemission spectroscopy to probe a strongly interacting Fermi gas. Nature 454, 744-747 (2008).

3. Eagles, D. M. Possible pairing without superconductivity at low carrier concentrations in bulk and thin-film superconducting semiconductors. Phys. Rev. 186, 456-463 (1969).

4. Leggett, A. J. Modern Trends in the Theory of Condensed Matter 13-27 (Springer, 1980).

5. Regal, C. M., Greiner, M. \& Jin, D. S. Observation of resonance condensation of fermionic atom pairs. Phys. Rev. Lett. 92, 040403 (2004).

6. Zwierlein, M. W. et al. Condensation of pairs of fermionic atoms near a Feshbach resonance. Phys. Rev. Lett. 92, 120403 (2004).

7. Chen, Q., Stajic, J. \& Levin, K. Applying BCS-BEC crossover theory to high temperature superconductors and ultracold atomic Fermi gases. Low Temp. Phys. 32, 406-423 (2006).

8. Perali, A., Pieri, P., Strinati, G. C. \& Castellani, C. Pseudogap and spectral function from superconducting fluctuations to the bosonic limit. Phys. Rev. B 66, 024510 (2002).

9. Ranninger, J. \& Robin, J. M. Manifestations of the pseudogap in the Boson-Fermion model for Bose-Einstein-condensation-driven superconductivity. Phys. Rev. B 53, R11961-R11963 (1996).

10. Tamai, A. et al. Strong electron correlations in the normal state of the iron-based $\mathrm{FeSe}_{0.42} \mathrm{Te}_{0.58}$ superconductor observed by angle-resolved photoemission spectroscopy. Phys. Rev. Lett. 104, 097002 (2010).

11. Nakayama, K. et al. Angle-resolved photoemission spectroscopy of the iron-chalcogenide superconductor $\mathrm{Fe}_{1.03} \mathrm{Te}_{0.7} \mathrm{Se}_{0.3}$ : Strong coupling behavior and the universality of interband scattering. Phys. Rev. Lett. 105, 197001 (2010).

12. Chen, F. et al. Electronic structure of $\mathrm{Fe}_{1.04} \mathrm{Te}_{0.66} \mathrm{Se}_{0.34}$. Phys. Rev. B 81, 014526 (2010).

13. Pourret, A. et al. Strong correlation and low carrier density in $\mathrm{Fe}_{1+y} \mathrm{Te}_{0.6} \mathrm{Se}_{0.4}$ as seen from its thermoelectric response. Phys. Rev. B 83, 020504 (2011).

14. Perali, A. et al. Evolution of the normal state of a strongly interacting Fermi gas from a pseudogap phase to a molecular Bose gas. Phys. Rev. Lett. 106, 060402 (2011).

15. Carlson, J. \& Reddy, S. Superfluid pairing gap in strong coupling. Phys. Rev. Lett. 100, 150403 (2008).

16. Schriefer, J. R. Theory of Superconductivity (ABC Westview Press, 1999).

17. Campuzano, J. C. et al. Direct observation of particle-hole mixing in the superconducting state by angle-resolved photoemission. Phys. Rev. B 53, R14737-R14740 (1996).

18. Liu, Z-H. et al. Unconventional superconducting gap in $\mathrm{NaFe}_{0.95} \mathrm{Co}_{0.05} \mathrm{As}$ observed by angle-resolved photoemission spectroscopy. Phys. Rev. B 84, 064519 (2011).

19. Randeria, M., Duan, J. \& Shieh, L. Bound states, Cooper pairing, and Bose condensation in two dimensions. Phys. Rev. Lett. 62, 981-984 (1989).

20. Liu, Y. \& Lin, C. T. A comparative study of $\mathrm{Fe}_{1+\delta} \mathrm{Te}_{1-x} \mathrm{Se}_{x}$ single crystals grown by Bridgman and self-flux techniques. J. Supercond. Nov. Magn. 24, 183-187 (2011).

\section{Acknowledgements}

We acknowledge useful discussions with $\mathrm{M}$. Randeria. This research was supported by the Israeli Science Foundation and by the E. and J. Bishop research fund.

\section{Author contributions}

Y.L. performed experiments and analysed data, E.L. performed experiments, K.C. prepared samples, Y.L., D.P. and A.K. wrote the paper.

\section{Additional information}

The authors declare no competing financial interests. Supplementary information accompanies this paper on www.nature.com/naturephysics. Reprints and permissions information is available online at www.nature.com/reprints. Correspondence and requests for materials should be addressed to A.K. 